\title{
LA MOSCA DOMÉSTICA COMO PORTADOR DE PATÓGENOS MICROBIANOS, EN CINCO CAFETERÍAS DEL NORTE DE BOGOTÁ
}

\section{THE DOMESTIC FLY AS CARRIER OF MICROBIAL PATHOGENS IN FIVE NORTHERN BOGOTA CAFETERIAS}

\author{
Johana Quiceno , Ximena Bastidas', Diana Rojas², Martín Bayona ${ }^{3}$
}

\begin{abstract}
1Estudiantes Facultad de Medicina. Universidad de Ciencias Aplicadas y Ambientales U.D.C.A. e-mail: quicenito9@gmail.com ${ }^{2}$ M.D. Especialista Epidemiología. Instituto Nacional de Salud - Facultad de Medicina, Universidad de Ciencias Aplicadas y Ambientales U.D.C.A. e-mail: dianarojasa@gmail.com ${ }^{3}$ Bacteriólogo. Especialista, M.Sc. Microbiología. Facultad de Medicina, Universidad de Ciencias Aplicadas y Ambientales U.D.C.A. Calle 222 No. 55-37, Bogotá, D.C. e-mail: mabayona@udca.edu.co: autor para correspondencia.
\end{abstract}

Rev. U.D.C.A Act. E Div. Cient. 13 (1): 23-29, 2010

RESUMEN

En el expendio público de alimentos en Colombia, se encuentra la frecuente interacción de la mosca y los humanos. Mundialmente, se conoce a este insecto, como vectores de agentes patógenos y con la concentración en áreas urbanas de las poblaciones humanas este riesgo se ha aumentado. Las moscas, se consideran importantes vectores de infecciones entéricas, que afectan a personas y a los animales domésticos. El presente trabajo pretendió establecer y demostrar los riesgos microbiológicos de ambientes en donde concurre la mosca doméstica. Se realizó un estudio descriptivo de corte transversal, en cinco cafeterías del norte de Bogotá. La población universo correspondió a las moscas presentes en los servicios de alimentación, de una zona del norte de Bogotá-Colombia, en las que se practicó análisis taxonómico y microbiológico, correspondiente a la determinación de enterobacterias, parásitos y hongos. Para la tabulación y el análisis de los datos, se emplearon Microsoft Excel y el programa estadístico SPSS versión 15. Los microorganismos aislados correspondieron: a bacterias, el 46,8\%, (Escherichia coli, Klebsiella pneumoniae); seguidos por parásitos, 31,9\% (Entamoeba histolytica y Blastocystis hominis) y hongos, 21,3\% (Aspergillus fumigatus y Criptococcus spp). El manejo de estos insectos, se debería considerar como otra medida de prevención en la enfermedad diarreica aguda.

Palabras clave: Insecto, restaurantes, microorganismos, diarrea, salud pública.

\section{SUMMARY}

In the public food sale establishments in Colombia the interaction between flies and humans is frequent. Globally, flies are known as vectors of pathogens and the concentration in urban areas of the human population has increased this risk. Flies are considered important vectors of enteric infections that affect people and pets. This work aimed to establish and demonstrate the microbiological risks of environments where the fly Musca domestica thrieves. A cross sectional study in five cafeterias in the northern Bogotá was performed. The population study implied M. domestica present in food services, where taxonomic and microbiological analysis were realized in order to identify Enterobacteriaceae, parasites and fungi. For tabulation and data analysis Microsoft Excel and SPSS version 15 were employed. The bacterial isolates accounted for $46.8 \%$ (Escherichia coli, Klebsiella pneumoniae), followed by parasites, $31.9 \%$ (Entamoeba histolytica and Blastocystis hominis) and fungi, 21.3\% (Aspergillus fumigatus and Cryptococcus spp.) The management of flies should be considered as another preventive measure in acute diarrheal disease.

Key words: Insect, restaurants, microorganisms, diarrhea, public health.

\section{INTRODUCCIÓN}

Mosca es el nombre genérico de un extenso grupo de especies de insectos pertenecientes al orden de los dípteros (Díptera). Se han clasificado unas 120.000 especies de dípteros, por 
ello, algunos científicos estiman que prevalece un millón de especies, donde una de cada diez especies de animales reconocidas por la ciencia es un díptero, existiendo muchas más variedades diferentes de dípteros, que de vertebrados (Graczyk et al. 2005).

Las organizaciones que regulan la sanidad y la salud pública han registrado 21 especies de moscas, como agentes causantes de enfermedades gastrointestinales, por su predilección por los ambientes contaminados y endofilia, es decir, tendencia a ingresar a edificaciones (Graczyk et al. 2005).

Musca domestica L. (Diptera: Muscidae) es atraída por diferentes sustratos: alimentos, desperdicios, secreciones y excretas para alimentarse, convirtiéndola en un vector mecánico eficiente de patógenos; éste insecto puede transportar microorganismos, a nivel externo, por la morfología de su cuerpo cubierto por setas o, internamente, en su tubo digestivo (Moissant et al. 2004) (Figura 1).

La presencia de moscas en zonas próximas a núcleos humanos representa un serio riesgo sanitario y medio ambiental. Las enfermedades más importantes transmitidas por las moscas a las personas y a los animales domésticos son intestinales e infecciones oculares (Bejar et al. 2006).

Las formas en que este insecto común puede transmitir los patógenos son: a) a través de su superficie corporal; b) por regurgitación de comida contaminada y c) por defecación de patógenos, siendo ésta vía muy importante, debido al efecto protector que le da el interior de su organismo al patógeno presente (Sasaki et al. 2000).

La fuente de origen de las infecciones microbianas, se relaciona, principalmente, con niveles bajos de higiene, de sanidad y la contaminación de comida. Estudios de laboratorio han demostrado que en la mosca común se pueden hallar más de cien agentes infecciosos para el hombre y para los animales, principalmente, aquellos causantes de diarrea (Ugbogu et al. 2006; Sasaki et al. 2000; Nunes, 2002; Manrique \& Delfín, 1997)

Responsabilizar a la $M$. domestica como vector mecánico de las enfermedades transmitidas por alimentos es difícil, ya que se sabe que el aislar patógenos demuestra una carga microbiana y no nos confirman si verdaderamente se lleva a cabo su transmisión. El aislar los microorganismos a partir de moscas es un índice potencial de contaminación (Arruma et al. 2005; Bejar et al. 2006).

Debido a lo anterior, se propuso en el presente estudio, demostrar que la continua interacción de las moscas con los alimentos, en este caso, en las cafeterías estudiadas, predispone a este tipo de riesgos microbiológicos, resultando la mosca ser un vector mecánico.

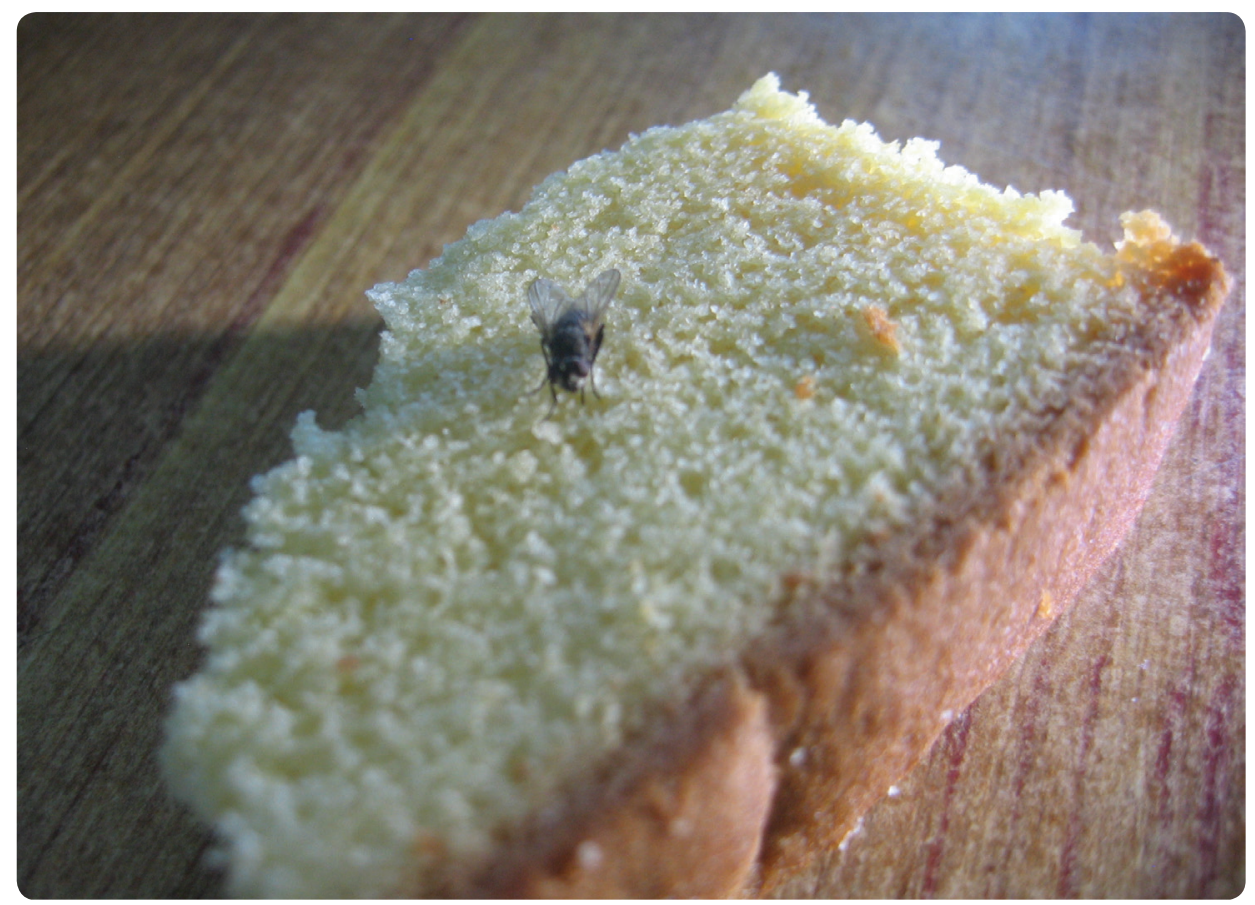

Figura 1. Mosca común, como vector mecánico presente en alimentos. 


\section{MATERIALES Y MÉTODOS}

Se realizó la selección de cinco establecimientos, que prestan el servicio de alimentación, ubicados en la norte de la ciudad de Bogotá. Los establecimientos, se encontraban en las siguientes coordenadas: Local 1: $4^{\circ} 48^{\prime} 01.94^{\prime \prime} \mathrm{N}$, 7402'54.13"'O; Local 2: 447'59.06”N, 7403'01.36”'O; Local

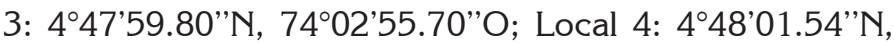
7402'54.10”O; Local 5: 448’01.64”N, 7402’50.66”O.

Se realizó un estudio descriptivo de corte transversal, tomando como población de estudio a diez moscas presentes en cada una de las cafeterías evaluadas, en el norte de Bogotá. Los respectivos insectos, se colectaron por medio de cintas atrapa moscas, las cuales, fueron ubicadas en el sitio específico donde se consumían los alimentos y que cumplen con los criterios de inclusión y no hacen parte de los criterios de exclusión.

Criterios de inclusión: Las moscas atrapadas en las cafeterías estudiadas del norte de Bogotá, retiradas del atrapa moscas y llevadas al laboratorio.

Criterios de exclusión: Moscas de otras especies; moscas que no pertenezcan a las cafeterías estudiadas en el norte de Bogotá y moscas que no tengan adecuada manipulación durante su recolección, es decir, que no estén en las cintas de las cafeterías estudiadas, o que no sean llevadas directamente al laboratorio para su estudio, con su respectiva rotulación

Los insectos fueron muestreados en cada local, a partir de diferentes sitios, teniendo en cuenta que cada uno de ellos tiene diversos métodos de control sanitario. Se realizó el análisis entomológico y la determinación taxonómica, llevada a cabo por la doctora Diana Rojas Álvarez.

El análisis microbiológico correspondió a la determinación de enterobacterias, parásitos y hongos. Este análisis, se procedió efectuando una siembra de la muestra (moscas obtenidas) en caldo BHI (infusión cerebro-corazón); posteriormente, se procedió a sembrar por agotamiento, el macerado de los insectos, en diferentes medios de cultivo bacteriano, como son Agar MacConkey (Agar selectivo para enterobacterias.) y agar Sabouraud (medio de cultivo recomendado para el aislamiento y desarrollo de hongos.); las cajas se incubaron durante 48 horas a $35^{\circ} \mathrm{C}$, para el grupo de las bacterias y, con relación a los hongos, a $25^{\circ} \mathrm{C}$, por tres a cinco días. Luego, se efectuaron diferentes tinciones, como coloración de Gram y preparación con azul de lactofenol. Para la identificación de los parásitos, se elaboraron preparaciones en fresco con solución salina y tinciones con lugol y Ziehl Neelsen. La identificación de bacterias (género y especie), se practicó por métodos automatizados, siguiendo las recomendaciones con el sistema BBL Crystal y el método de REMEL para bacterias entéricas, los cuales, son micro-métodos cualitativos que emplean sustratos convencionales y cromogénicos (Murray et al. 2002; Prats, 2006).

Para la identificación de las levaduras, se emplearon pruebas de asimilación y de fermentación de carbohidratos, así como características microscópicas y macroscópicas en agar Sabouraud; para la identificación de hongos filamentosos, se describieron características microscópicas y macroscópicas (Alexoupoulus \& Mims, 1985; Bonifaz, 2010).

Estadística: Se realizó una estadística cualitativa, donde se evaluó Chi-cuadrado. Para la tabulación y el análisis de los datos, se empleó Microsoft Excel y el programa estadístico SPSS versión 15, en español.

\section{RESULTADOS Y DISCUSIÓN}

Todos los insectos colectados y evaluados presentaron las características de la especie Musca domestica (Figura 2).

Los microorganismo aislados fueron respectivamente: bacterias (46,8\%), como Citrobacter freundii, Klebsiella pneumoniae, K. oxytoca, Serratia marcenscens, Escherichia coli, Enterobacter cloacae, E. aerogenes, E. gergoviae, Salmonella spp., Acinetobacter calcoaceticus, Yersinia enterocolitica, Y. intermedia y Providencia rettgeri (Tabla 1); parásitos (31,9\%), como: Entamoeba coli, E. histolytica, E. hartmanii, E. dispar, Endolimax nana, Blastocystis hominis, Iodamoeba butschlii (Tabla 2) y hongos (21,3\%), como: Candida sp., Aspergillus fumigatus, Penicillium sp, Rhizopus sp, Criptococcus sp. y Fusarium sp. (Tabla 3). Estos datos coinciden, parcialmente, con los trabajos reportados por Bejar et al. 2006, quienes demostraron que la M. domestica es un vector mecánico de bacterias enteropatógenas, en distritos de Lima y de Callao, Perú, donde a partir de 780 moscas domésticas, se aisló $E$. coli enteropatógena (ECEP), Salmonella typhi, Shigella flexneri y Y. enterocolitica. Así mismo, Arruma et al. (2005) describen la presencia de $Y$. enterocolitica y S. typhi aisladas, a partir de M. domestica. Fotedar et al. (1992) refieren a la mosca, como un vector mecánico de K. pneumoniae, en centros hospitalarios.

M. domestica ha sido confirmada como un eficiente vector de E. coli, específicamente, la cepa O 157: H7. Esta cepa puede ser transportada en el tubo digestivo, multiplicarse en las piezas bucales y luego ser excretada por tres días consecutivos (Moissant et al. 2004; Sasaki et al. 2000). La infección por $E$. coli 0157:H7, se asocia con el síndrome urémico hemolítico, una enfermedad que se caracteriza por la destrucción de los glóbulos rojos sanguíneos, una drástica reducción de las plaquetas y una insuficiencia renal aguda (Murray et al. 2002). 


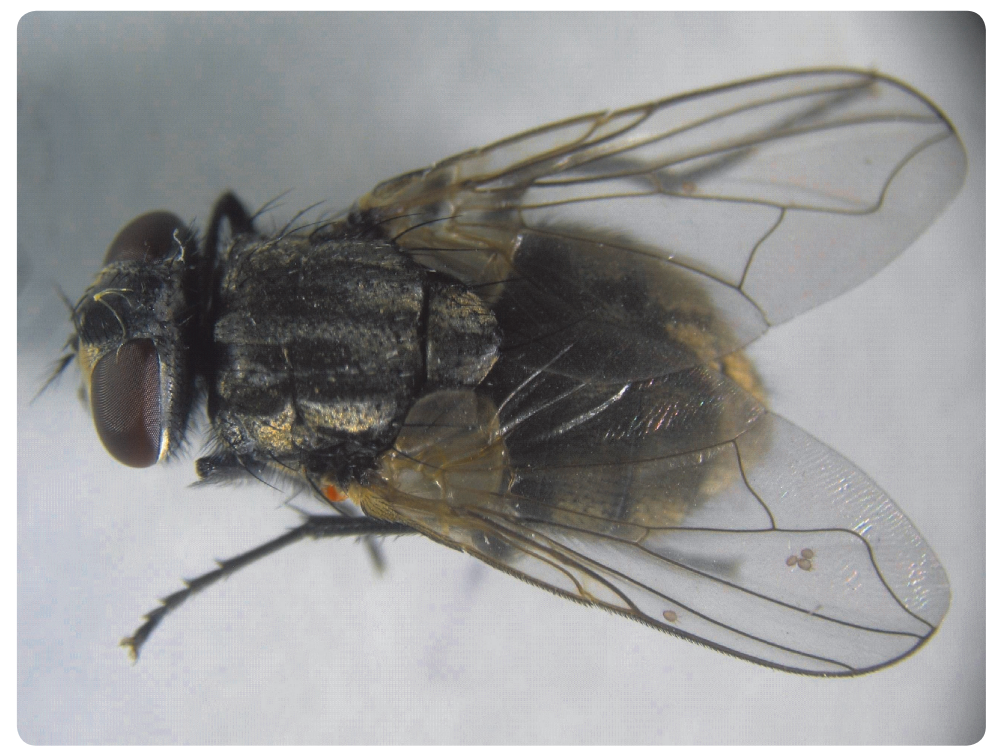

Figura 2. Espécimen de Musca domestica L. (Diptera: Muscidae) colectado en una de las cafeterías evaluadas.

E. coli fue encontrada en tres de los cinco locales estudiados. No se registró dependencia entre el local y los microorganismos, como bacterias y parásitos. En 2004, Alam \& Zurek evidenciaron la presencia de E. coli O157:H7 en altas concentraciones en $M$. doméstica en una granja, especialmente, durante el verano. En Venezuela, de igual manera, se realizó un estudio en $M$. doméstica, donde Moissant (2004) detectó E coli, como principal patógeno y, en Lima, Perú, Bejar et al. (2006) realizaron un estudio a partir de moscas presentes en mercados y en basurales, hallando E. coli, Salmonella spp., Shigella spp. y Yersinia spp. Los géneros bacterianos, anteriormente mencionados, están incluidos en la familia Enterobacteriacea, los cuales, son un grupo de heterogéneo de bacilos cortos Gram negativos, cuyo hábitat es el intestino de humanos y de animales, asociados con enfermedades, en donde las pobres condiciones sanitarias contribuyen con la alta frecuencia de

Tabla 1. Bacterias encontradas en las cinco cafeterías evaluadas.

\begin{tabular}{|c|c|c|c|c|c|}
\hline MICROORGANISMO & LOCAL 1 & LOCAL 2 & LOCAL 3 & LOCAL 4 & LOCAL 5 \\
\hline Citrobacter freundii & $\mathrm{X}$ & $\mathrm{X}$ & & & \\
\hline Klebsiella pneumoniae & $\mathrm{X}$ & $\mathrm{X}$ & $\mathrm{X}$ & & \\
\hline Serratia marcenscens & $\mathrm{X}$ & $\mathrm{X}$ & & $\mathrm{X}$ & $\mathrm{X}$ \\
\hline Escherichia coli & $\mathrm{X}$ & & $\mathrm{X}$ & & $\mathrm{X}$ \\
\hline Providencia rettgeri & & $\mathrm{X}$ & & & \\
\hline Enterobacter cloacae & & $\mathrm{X}$ & & $\mathrm{X}$ & \\
\hline Enterobacter aerogenes & & & $\mathrm{X}$ & & \\
\hline Acinetobacter calcoaceticus & & & & $\mathrm{X}$ & \\
\hline Salmonella sp. & & & & $\mathrm{X}$ & $\mathrm{X}$ \\
\hline Klebsiella oxytoca & & & & & $\mathrm{X}$ \\
\hline Enterobacter gergoviae & & & & & $\mathrm{X}$ \\
\hline Yersinia enterocolitica & & & & & $\mathrm{X}$ \\
\hline Yersinia intermedia & & & & & $\mathrm{X}$ \\
\hline
\end{tabular}


Tabla 2. Parásitos hallados en las cinco cafeterías evaluadas.

\begin{tabular}{|c|c|c|c|c|c|}
\hline MICROORGANISMO & LOCAL 1 & LOCAL 2 & LOCAL 3 & LOCAL 4 & LOCAL 5 \\
\hline Entamoeba coli & $\mathrm{X}$ & & & $\mathrm{X}$ & $\mathrm{X}$ \\
\hline Entamoeba histolytica-E. dispar & $\mathrm{X}$ & & $\mathrm{X}$ & & $\mathrm{X}$ \\
\hline Endolimax nana & $\mathrm{X}$ & & $\mathrm{X}$ & & $\mathrm{X}$ \\
\hline Blastocystis hominis & & $\mathrm{X}$ & $\mathrm{X}$ & & $\mathrm{X}$ \\
\hline lodamoeba butschlii & & $\mathrm{X}$ & & & \\
\hline Entamoeba hartmanii & & $\mathrm{X}$ & & $\mathrm{X}$ & \\
\hline
\end{tabular}

Tabla 3. Hongos encontrados en las cinco cafeterías evaluadas.

\begin{tabular}{|c|c|c|c|c|c|}
\hline MICROORGANISMO & LOCAL 1 & LOCAL 2 & LOCAL 3 & LOCAL 4 & LOCAL 5 \\
\hline Aspergillus fumigatus & & & $\mathrm{X}$ & $\mathrm{X}$ & $\mathrm{X}$ \\
\hline Penicillium sp. & & & & & $\mathrm{X}$ \\
\hline Rhizopus sp. & & & & $\mathrm{X}$ & $\mathrm{X}$ \\
\hline Criptococcus sp. & & & & $\mathrm{X}$ & $\mathrm{X}$ \\
\hline Candida sp. & & & & & $\mathrm{X}$ \\
\hline Fusarium sp. & & & & & $\mathrm{X}$ \\
\hline
\end{tabular}

gastroenteritis, en muchas regiones del mundo (Restrepo et al. 2003; Moriya et al. 1999).

Salmonella spp. es un microorganismo causante de gastroenteritis, asociado con enfermedades transmitidas por alimentos. Este microorganismo fue aislado en dos de los cinco locales estudiados en el presente trabajo. Es probable que la fuente de inoculo esté relacionado con el ambiente cercano de los expendios de alimentos a la presencia en los alrededores de vacunos, de equinos y de caninos. Ugbogu et al. (2006) realizó un estudio en $M$. domestica aislando Salmonella spp. en un $61,7 \%$ del total de las moscas evaluadas, al igual que Shigella que, en este caso, fue hallado en un $100 \%$ de las moscas. La tasa de infección de las moscas con especies de Shigella observada en este estudio fue muy alta y hace un llamamiento para un control correcto mecánico de las moscas, saneamiento ambiental y el uso de insecticidas, si es del caso.

Forster et al. (2009) encontraron, en aislamientos realizados a partir del contenido intestinal de $M$. doméstica, diferentes microorganismos patógenos, como E. coli, Campylobacter spp., S. aureus, Enterococcus spp., K. oxytoca y P. mirabilis, recolectadas de localidades rurales (concretamente un establo de ganado, una perrera, un establo de caballos y un chiquero). Estos datos son concordantes con el presente trabajo, si se tiene en cuenta que en los alrededores de los servicios de alimentación, concurren en una zona campestre, rodeados por diversos animales (equinos, vacunos, porcinos y caninos).

La transmisión de bacterias patógenas y parásitos aunque no es probada en el presente estudio, es el más posible factor de riesgo del contacto de $M$. domestica con el hombre, especialmente, con sus alimentos. El ser portador de microorganismos, en su mayoría patógenos, es una situación que nos pone a pensar en las diferentes estrategias, para el manejo de alimentos, recolección de desechos y manejo de estiércol, como lo afirman Manrique E Delfín (1997) y Bejar et al. (2006).

Dentro de los parásitos registrados, se tiene $E$. histolytica $-E$. dispar, B. hominis y protozoarios comensales, lo cual, concuerda parcialmente con el trabajo reportado por Cárdenas \& Martínez (2004), quienes a partir de moscas procesadas con agua destilada estéril, para obtener un macerado intestinal, hallaron parásitos intestinales, tales como B. hominis, G. lamblia, Criptosporidium spp., C. cayetanensis, Iodamoeba butschlii, E. nana y Chilomastix mesnilii, confirmando que esta especie actúa como vector mecánico de parásitos, ya sea por sus características biológicas, sus hábitos y por el hecho de encontrar muestras positivas con quistes de protozoarios, de importancia clínica; E. histolytica es un protozoario patógeno intestinal definido por la literatura mundial. En cuanto a la presencia de parásitos intestinales comensales, su presencia carece de importancia clínica, pero tiene importancia epidemiológica, 
ya que es catalogado como un referente de la contaminación con materia fecal. $B$. hominis es reconocido como uno de los parásitos más prevalentes, con un poder patógeno controvertido, como lo afirma (Zerpa ETerashima, 2000).

En el presente trabajo, no se observó la presencia de huevos del grupo de los helmintos. De acuerdo con Graczyk et al. (2005), las moscas son frecuentemente portadoras de estos parásitos. La participación de estos insectos en la transmisión de los parásitos es de alto riesgo, si se manifiesta en hospitales, donde Toxoplasma gondii tiene gran importancia en pacientes inmunosuprimidos (Fotedar et al. 1992)

Castillo et al. (2008) determinaron, a partir de macerados de $M$. domestica obtenidas de una plaza de mercado, la presencia de parásitos, como E. nana, B. hominis, E. hartmanni, E. coli, C. cayetanensis, G. lamblia y T. cannis. Estos datos concuerdan, parcialmente, con el presente estudio, ya que $C$. cayetanensis y $T$. cannis no fueron reportados. Los hongos hallados en las moscas colectadas en este estudio correspondieron a Fusarium spp., Penicillium spp., Aspergillus fumigatus, Rhizopus spp., Candida spp. y Criptococcus spp. De igual manera, se encontró dependencia entre estos hongos y el local evaluado, destacando que en el local 5, se localizó mayor cantidad de estos microorganismos, lo cual, refleja que este local registra gran contaminación y falta de higiene, de acuerdo con lo observado inicialmente (Cuadro 1). Estos hongos son reportados en la literatura como patógenos oportunistas, sobre todo en pacientes inmunocomprometidos (Bonifaz, 2010; Nunes, 2002; Tipismana et al. 2005).

Cuadro 1. Local 5 y su dependencia frente a los hongos encontrados.

\begin{tabular}{|l|c|c|}
\hline \multicolumn{2}{|c|}{} & Hongos \\
\hline \multirow{4}{*}{ Local 5 } & Chi-cuadrado & 22,761 \\
\cline { 2 - 3 } & Grados de libertad & 4 \\
\cline { 2 - 3 } & Significancia. &, $000\left(^{*}, \mathrm{a}\right)$ \\
\hline
\end{tabular}

Los hongos hallados en el presente trabajo concuerdan con el estudio realizado por Nunes (2002), ya que los microorganismos aislados e identificados, a partir de Mosca domestica en común, correspondieron a Penicillium spp., Fusarium spp., Rhizopus spp., A. fumigatus y Criptococcus spp. y Candida spp., a diferencia de Alternaria spp., Curvularia spp. y micelio estéril, que no fueron reportados en esta investigación. Así mismo, Forster et al. (2009), en un estudio llevado a cabo, a partir del contenido intestinal de $M$. domestica obtenida de sectores rurales, identificó $A$. fumigatus, C. albicans y C. tropicalis.

De acuerdo con la carga microbiana por restaurantes evaluados, se registró a la cafetería 5 con el $34 \%$ de todos los microorganismos aislados siguiéndolo en orden la 4, con $19 \%$, el local 3 , con $17 \%$ y las cafeterías 1 y 2 con el $14,9 \%$, cada uno, lo cual, se ve determinado, directamente, por el bajo nivel de aseo de estos puntos y la presencia de animales a sus alrededores, lo que atrae a estos insectos. Con los resultados obtenidos, se demuestra, una vez más, la necesidad de implementar campañas en sanidad ambiental y en educación, no solo en manipuladores de alimentos sino en la población en general, en temas como hábitos de higiene y en tratamiento y manejo de los desechos sólidos y excretas de humanos y de animales.
Agradecimientos. Los autores agradecen por la colaboración prestada a la Universidad de Ciencias Aplicadas y Ambientales U.D.C.A, directivas, a la doctora María Teresa Ríos Tovar, así mismo a los restaurantes participantes en el desarrollo de la presente investigación. Conflicto de intereses: Los autores declaramos que no existen conflictos de intereses que pongan en riesgo la validez de los resultados presentados. Financiación: El trabajo fue financiado por los autores del presente trabajo, en colaboración con el laboratorio de Microbiología de la Universidad de Ciencias Aplicadas y Ambientales U.D.C.A.

\section{BIBLIOGRAFÍA}

1. ALAM, M.; ZUREK, L. 2004. Association of Escherichia coli O 157: $\mathrm{H} 7$ with houseflies on a cattle farm. Appl. Environ. Microbiol. 70(12):7578-7580.

2. ALEXOPOULOS, C.; MIMS, C. 1985. Introducción a la micología. $1^{a}$ Ed. Ed. Omega, Barcelona (España). 252p.

3. ARRUMA, N.; GHENGHESH, K.; BEN AISSA, R.; ELAMARI, A. 2005. Carriage by the housefly (Musca domestica) of multiple-antibiotic resistant bacteria that are po- 
tentially pathogenic to humans, in hospital and other urban environments in Misurata, Lybia. Ann. Trop. Med. Parasitol. 99(8):795-802.

4. BEJAR, V.; CHUMPITAZ, J.; PAREJA, E.; VALENCIA, E.; HUAMÁN, A.; SEVILLA, C.; TAPIA, M.; SAEZ, G. 2006. Musca domestica como vector mecánico de bacterias entero patógenas en mercados y basurales de Lima y Callao. Rev. Perú Med. Exp. Salud Pública. 23(1):39-43.

5. BONIFAZ, A. 2010. Micología Médica básica. $3^{a}$ Ed. Mc Graw Hill. p.280-375.

6. CÁRDENAS, M.; MARTÍNEZ, R. 2004. Protozoarios parásitos de importancia en Salud Pública transportados por M. domestica Linnaeus en Lima, Perú. Rev. Perú Biol. 11(2):149-152.

7. CASTILLO, C.; CASTRO, M.; CARHUAPOMA, C.; CASTRO, H.; CASTRO, R.; CHAMBI, J. 2008. Parásitos de importancia en salud pública transportados por $M$. domestica. Lima-Perú. CIMEL. 13(2):49-53.

8. FORSTER, M.; SIEVERT, K.; MESSLER, S.; KLIMPEL, S.; PFEFFER, K. 2009. Comprehensive study on the occurrence and distribution of pathogenic microorganisms carried by synanthropic flies caught at different rural locations in Germany. J. Med. Entomol. 46(5):1164-1166.

9. FOTEDAR, R.; BANERJEE, U.; SAMANTRY, J.; SHINIWA, U. 1992. Vector potential of hospital houseflies with special reference to Klebsiella species. Epidemiol. Infect. 109(1):143-147.

10. GRACZYK, T.; KNIGHT, R.; TAMANG, L. 2005. Mechanical transmission of human protozoan parasites by insects. Clinic. Microbiol. Rev. 18(1):128-132.

11. MANRIQUE, P.; DELFÍN, H. 1997. Importancia de las moscas como vectores potenciales de enfermedades diarreicas en humanos. Biomédica. 8:163-170.

12. MOISSANT, E.; TKACHUK, O.; ROMAN, R. 2004. Detección de agentes bacterianos en adultos de Musca domestica (Diptera: Muscidae) recolectadas en Maracay, Estado Aragua, Venezuela. Entomotrópica. 19(3):161-164.
13. MORIYA, K.; FUJYBAYASHI, T.; YOSHIHARA, A.; MATSUDA, N.; SUMI, N.; UMEZAKI, H.; KURAHASHI, N.; AQUI, A.; WADA, H.; WATANABE, A. 1999. Verotoxinproducing Escherichia coli O157:H7 carried by the housefly in Japan. Med. Vet. Entomol. 13:214-216.

14. MURRAY, P.; ROSENTHAL, K.; KOAYASHI, G.; PFALLER, M. 2002. Medical Microbiology. 4a Ed. Mosby Inc. (Elsevier Science) St. Luis, Missouri. p.175-426.

15. NUNES, M. 2002. Isolation of fungi in Musca domestica Linnaeus, 1758 (Diptera: Muscidae) captured at two natural breeding grounds in the municipality of Seropedica, Rio de Janeiro, Brazil. Mem Inst. Oswaldo Cruz, Rio de Janeiro. 97(8):1107-1110.

16. PRATS, G. 2006. Microbiología Clínica. Ed. Médica Panamericana, S.A. p.39-47.

17. RESTREPO, A.; ROBLEDO, J.; LEIDERMAN, E.; RESTREPO, M.; BOTERO, D.; BEDOYA, V. 2003. Fundamentos de Medicina -Enfermedades Infecciosas. $6^{a}$ Ed. Corporación para investigaciones Biológicas. p.445-455.

18. SASAKI, T.; KOBAYASHI, M.; AGUI, N. 2000. Epidemiological potential of excretion and regurgitation by Musca domestica (Diptera: Muscidae) in the dissemination of Escherichia coli 0157: H7. Food. J. Med. Entomol. 37(6):945-949.

19. TIPISMANA, C.; ASTUDILLO, L.; GUILLERMO, J. 2005. Hongos de importancia agrícola presentes en moscas de la fruta del valle de Ica, Perú. Rev. Perú Biol. 12(3):1-11.

20. UGBOGU, O.; NWACHUKWV, N.; OGBUAGU, V. 2006. Isolation of Salmonella and Shigella species from house flies (Musca domestica) in Uturu, Nigeri. Afr. J. Biotech. 5(11):1090-1091.

21. ZERPA, R.; TERASHIMA, A. 2000. Blastocystosis. Diagnóstico. 39(3):120-121.

Recibido: Julio 14 de 2010

Aceptado: Octubre 21 de 2010 